\title{
Pleural effusion as an atypical presentation of Kawasaki disease: a case report and review of the literature
}

\author{
Elif Arslanoglu Aydin', Selcan Demir ${ }^{2}$, Orkun Aydin ${ }^{3}$, Yelda Bilginer $^{2}$ and Seza Ozen ${ }^{2 *}$
}

\begin{abstract}
Background: Kawasaki disease is an acute, febrile vasculitis of childhood that affects medium-sized arteries, predominantly the coronary arteries. It is a multisystem disease; therefore, it may present with non-cardiac findings of disease.

Case presentation: Here, we report the case of 7-year-old Turkish girl who presented with symptoms of fever, chest pain, and vomiting, who was diagnosed as having Kawasaki disease. We also present a literature review on pulmonary involvement due to Kawasaki disease.

Conclusion: Pediatricians should consider the diagnosis of Kawasaki disease in the presence of pneumonia and pleural effusion that is nonresponsive to antibiotic therapy. This will prevent delay in diagnosis and the adverse consequences of the disease.
\end{abstract}

Keywords: Kawasaki disease, Pleural effusion, Pulmonary involvement

\section{Background}

Kawasaki disease (KD) is one of the most common vasculitis disorders of childhood [1]. Although it is a multisystem disease that mainly affects the coronary arteries, it can, rarely, present with unusual system involvement of the pulmonary system, gastrointestinal tract, central nervous system, and genitourinary system [1]. Here, we report the case of a patient with KD who presented with an unusual form of pleural effusion. We also present a literature review on the subject.

\section{Case presentation}

A 7-year-old Turkish girl presented to a local hospital with fever, chest pain, and vomiting. At hospital admission, she was febrile with a respiratory rate of 50 per minute. On physical examination, auscultation of her lungs revealed diminished breath sounds of the lower lobe of her left lung. An anteroposterior (AP) chest X-ray and chest ultrasonography showed a left lower lobar consolidation with minimal pleural effusion. She was

\footnotetext{
* Correspondence: sezaozen@gmail.com

${ }^{2}$ Department of Pediatric Rheumatology, Hacettepe University Faculty of Medicine, 06100 Ankara, Turkey

Full list of author information is available at the end of the article
}

hospitalized and sulbactam ampicillin (SAM), ceftriaxone, and clarithromycin were initiated. On the third day, her condition worsened with increasing pleural effusion (Fig. 1). Thoracentesis was performed. SAM and ceftriaxone treatments were discontinued and meropenem and vancomycin were started. A chest tube was inserted and $130 \mathrm{~mL}$ of pus was drained. Light's criteria were positive for an exudative pleural effusion; a pleural fluid culture was sterile. After 4 days, the chest tube was removed. High fever persisted for 15 days despite broad spectrum antibiotics, and acute-phase reactants remained high; therefore, she was referred to our hospital for further evaluation.

She had a fever with a temperature of $38.1^{\circ} \mathrm{C}$, her respiratory rate was $48 /$ minute, heart rate was $125 /$ minute, blood pressure was $90 / 65 \mathrm{~mm} \mathrm{Hg}$, and oxygen saturation was $95 \%$. A physical examination revealed non-purulent conjunctivitis in both eyes, perianal peeling, and periungual desquamation on her hand, fingers, and toes. All other findings in the physical examination were unremarkable. She had unilateral cervical lymphadenopathy and a rash on her extremities while in the other hospital. Her past medical history was unremarkable, as was her

(c) The Author(s). 2019 Open Access This article is distributed under the terms of the Creative Commons Attribution 4.0 International License (http://creativecommons.org/licenses/by/4.0/), which permits unrestricted use, distribution, and reproduction in any medium, provided you give appropriate credit to the original author(s) and the source, provide a link to the Creative Commons license, and indicate if changes were made. The Creative Commons Public Domain Dedication waiver (http://creativecommons.org/publicdomain/zero/1.0/) applies to the data made available in this article, unless otherwise stated. 


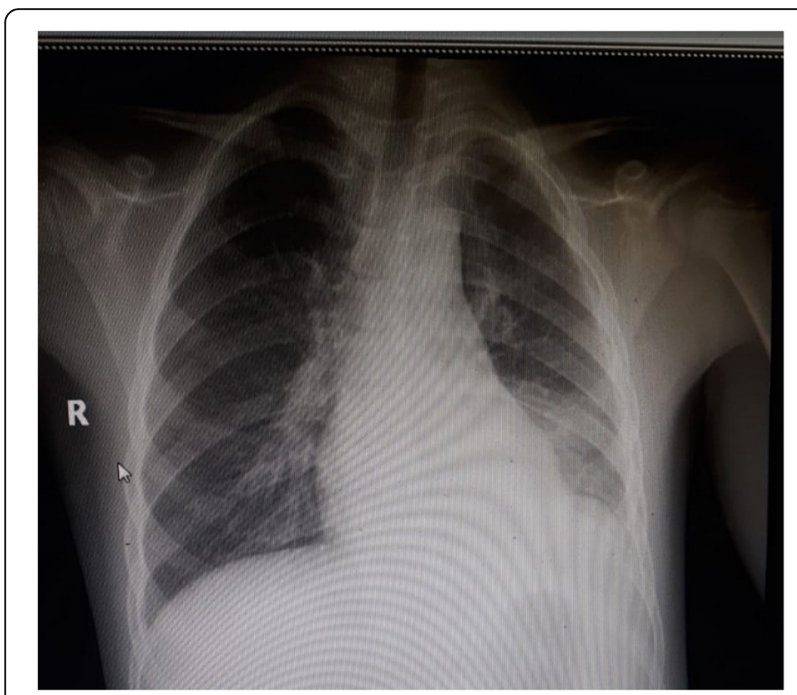

Fig. 1 Chest $X$-ray of the patient showing left lower lobar consolidation with pleural effusion

family history. Immunizations were up-to-date for her age.

On admission to our hospital, the laboratory findings were as follows: hemoglobin $10.2 \mathrm{~g} / \mathrm{dL}$, white blood cells $14,000 / \mu \mathrm{L}$, and platelets $736,000 / \mu \mathrm{L}$. C-reactive protein (CRP) was $4.26 \mathrm{mg} / \mathrm{dL}$ (normal, $0-0.8 \mathrm{mg} / \mathrm{dL}$ ), the erythrocyte sedimentation rate (ESR) was $42 \mathrm{~mm}$ /hour (normal, 0-20 mm/hour), and the albumin, creatinine,

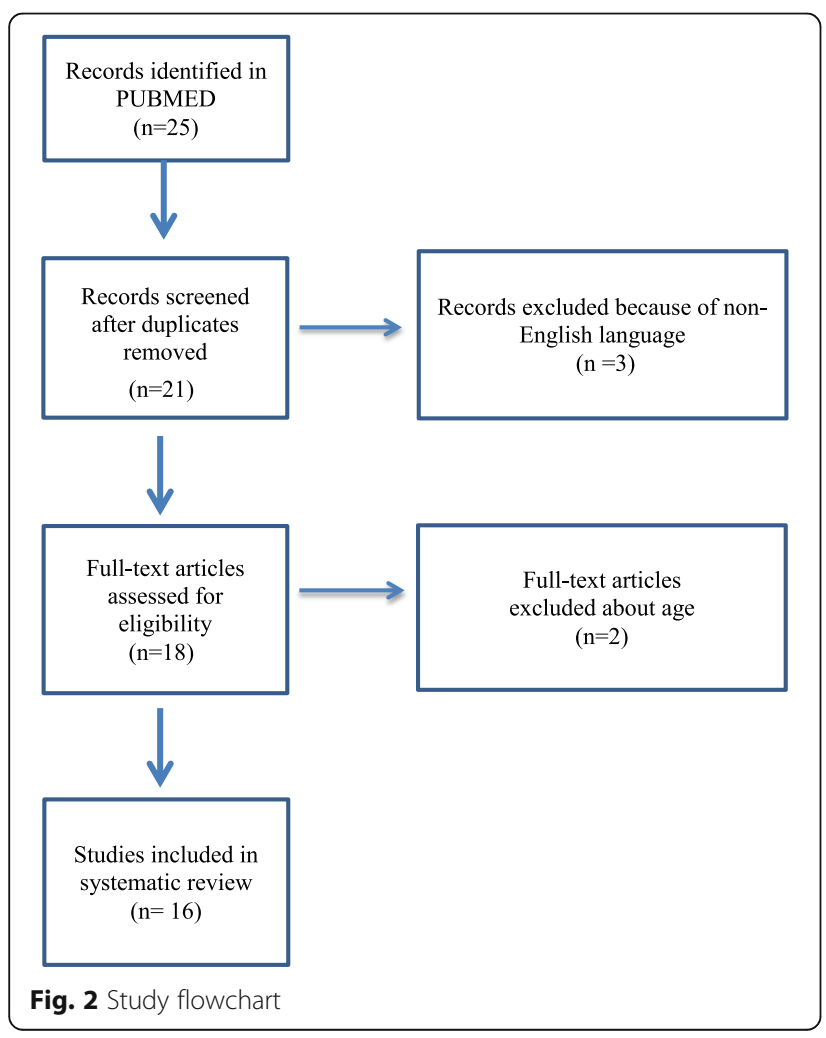

aspartate aminotransferase, alanine aminotransferase, gamma glutamyl transferase, blood urea nitrogen, calcium, sodium, chloride, and potassium levels were normal. Urine analysis was normal.

A chest X-ray was normal. Perivascular brightness and echogenicity of her right coronary artery was noted on transthoracic echocardiography (TTE). She was diagnosed as having KD based on the presence of fever, bilateral non-purulent conjunctivitis, cervical adenopathy, perianal peeling, periungual desquamation, elevated acute-phase reactants (ESR, CRP), thrombocytosis, and coronary artery involvement (CAI). Intravenous immunoglobulin (IVIG) $(2 \mathrm{~g} / \mathrm{kg}$, infusion in 12 hours) and acetylsalicylic acid $(60 \mathrm{mg} / \mathrm{kg}$ per day) were initiated. The fever resolved after IVIG infusion. At a 3-month follow-up visit, the acute-phase reactants and a TTE were normal. One year after the diagnosis, a TTE was normal and she was perfectly healthy.

\section{Discussion and conclusion}

The most important complication of KD is CAI, which leads to enlargement, aneurysm, ischemic heart disease, and sudden death [1]. The clinical course of KD is highly variable. There are no pathognomonic clinical or laboratory findings to help diagnose KD. The diagnosis of KD in this case was made using the criteria of the American Heart Association [1]. In the presence of at least 5 days of fever, if there are at least four of the five principal criteria (cervical adenopathy, bilateral non-purulent conjunctivitis, oropharyngeal mucosal changes, polymorphous rash, erythema of the palms or soles, and edema of the hands or feet) the patient is diagnosed as having $\mathrm{KD}[1]$.

KD may present with uncommon symptoms such as pneumonia, pleural effusion, diarrhea, vomiting, sterile pyuria, gallbladder hydrops, acute cholestatic hepatitis, arthritis, and aseptic meningitis [2-7]. Pulmonary system involvement of KD is very rare; KD can present as pneumonia, pulmonary nodules, bronchopneumonia, hydropneumothorax, and pleural effusion $[6,8,9]$. Singh et al. showed that $1.3 \%$ of patients had pulmonary involvement and pleural effusion was seen in $54.5 \%$ of these patients [6]. Ugi et al. reported the case of an adult patient who presented with pulmonary involvement, specifically bilateral massive pleural effusions [10]. Occasionally, pleural effusion may be associated with bacterial agents such as Mycoplasma pneumoniae and Streptococcus [11, 12]. Pulmonary symptoms are mostly initially treated with antibiotics. However, if fever and accompanying signs ensue, the diagnosis of KD should be considered. Patients with pulmonary involvement may be more likely to have CAI due to delays in diagnosing $\mathrm{KD}$ and administration of IVIG [12-17]. 
Table 1 Clinical symptoms and laboratory parameters of patients who had pulmonary involvement associated with Kawasaki disease

\begin{tabular}{|c|c|c|c|c|c|c|c|c|c|c|c|}
\hline $\begin{array}{l}\text { Authors, year, } \\
\text { reference number }\end{array}$ & Rash & $\begin{array}{l}\text { Oral } \\
\text { changes }\end{array}$ & $\begin{array}{l}\text { Extremity } \\
\text { changes }\end{array}$ & $\begin{array}{l}\text { Red } \\
\text { eyes }\end{array}$ & Adenitis & Other clinical symptoms & $\begin{array}{l}\mathrm{Hb} \\
(\mathrm{g} / \\
\mathrm{dL})\end{array}$ & $\begin{array}{l}\text { WBC } \\
\left(/ \mathrm{mm}^{3}\right)\end{array}$ & $\begin{array}{l}\text { Plt } \\
\left(/ \mathrm{mm}^{3}\right)\end{array}$ & $\begin{array}{l}\text { CRP } \\
(\mathrm{mg} / \\
\mathrm{dL})\end{array}$ & $\begin{array}{l}\text { ESR } \\
\text { (mm/ } \\
\text { hour) }\end{array}$ \\
\hline Singh et al., 2018, [6] & $5^{a}$ & $1^{a}$ & $8^{a}$ & $1^{a}$ & $0^{a}$ & $\begin{array}{l}\text { Perianal desquamation, } 3^{a} \text {; } \\
\text { irritability, } 1^{a}\end{array}$ & NA & $25,009^{b}$ & $886,545^{b}$ & $14.05^{c}$ & $53.75^{d}$ \\
\hline $\begin{array}{l}\text { Alhammadi and } \\
\text { Hendaus, 2013, [12] }\end{array}$ & NA & NA & NA & NA & NA & NA & NA & 24,000 & 600,000 & 10 & 65 \\
\hline Lee et al., 2011, [11] & NA & NA & NA & NA & NA & NA & NA & NA & NA & NA & NA \\
\hline Lee et al., 2010, [14] & Yes & Yes & Yes & Yes & Yes & Perianal desquamation & NA & 5500 & 178,000 & 2.8 & 21 \\
\hline Falcini et al., 2009, [15] & No & No & No & Yes & No & Irritability & 9.5 & 21,800 & 710,000 & 27.8 & 99 \\
\hline $\begin{array}{l}\text { Elizabeth et al., } \\
\text { 2007, [16] }\end{array}$ & No & Yes & Yes & No & Yes & Irritability & 10.2 & 12,800 & 550,000 & 7.7 & 86 \\
\hline Yavuz et al., 2007, [17] & Yes & Yes & Yes & Yes & No & $\begin{array}{l}\text { Non-pigmented keratic } \\
\text { precipitates in both of the } \\
\text { patient's eyes, sterile pyuria }\end{array}$ & 10.4 & 32,800 & 734,000 & 21.8 & 90 \\
\hline $\begin{array}{l}\text { Sittiwangkul and } \\
\text { Pongprot, 2004, [13] }\end{array}$ & Yes & No & Yes & Yes & No & Irritability & NA & 21,200 & 231,000 & 4.77 & 66 \\
\hline $\begin{array}{l}\text { de Magalhães et al., } \\
\text { 2012, [21] }\end{array}$ & Yes & Yes & Yes & Yes & No & $\begin{array}{l}\text { Induration at the BCG site, } \\
\text { perianal desquamation }\end{array}$ & 6.5 & 25,000 & 905,000 & 34 & 120 \\
\hline $\begin{array}{l}\text { Hamada et al., } \\
\text { 2005, [18] }\end{array}$ & Yes & Yes & Yes & No & Yes & Hepatomegaly & NA & 17,800 & NA & 13.9 & NA \\
\hline $\begin{array}{l}\text { D'Souza et al., } \\
\text { 2006, [20] }\end{array}$ & No & No & Yes & No & Yes & Sterile pyuria & 9.8 & 56,800 & 690,000 & NA & 138 \\
\hline \multicolumn{12}{|l|}{ de Maddi et al., 2009, [22] } \\
\hline Case 1 & No & Yes & No & Yes & Yes & No & 8.7 & 11,200 & 561,000 & 10.4 & 70 \\
\hline Case 2 & No & No & No & No & No & No & 9 & 26,960 & 142,000 & 40.8 & 107 \\
\hline Case 3 & Yes & Yes & No & Yes & No & Irritability, sterile pyuria & 11 & 18,500 & $1,087,000$ & 2.95 & 50 \\
\hline \multicolumn{12}{|l|}{ Freeman et al., 2003, [23] } \\
\hline Case 1 & Yes & Yes & No & Yes & No & Irritability & NA & NA & $1,120,000$ & NA & NA \\
\hline Case 2 & No & Yes & No & Yes & Yes & Torticollis & NA & NA & $1,102,000$ & NA & 114 \\
\hline Case 3 & Yes & No & No & No & Yes & Anorexia & NA & NA & 450,000 & 10.5 & NA \\
\hline \multicolumn{12}{|c|}{ Kobayashi et al., 2006, [24] } \\
\hline Case 1 & Yes & Yes & No & Yes & Yes & & 9.6 & 13,000 & 321,000 & 12.6 & 102 \\
\hline Case 2 & Yes & Yes & Yes & Yes & No & Induration at the BCG site & 11.6 & 18,800 & 314,000 & 7.6 & NA \\
\hline Vaidya et al., 2017, [8] & Yes & Yes & Yes & No & No & & 8.9 & 15,600 & 567,000 & 5.6 & 40 \\
\hline \multicolumn{12}{|l|}{ Akagi et al., 2017, [19] } \\
\hline Case 1 & Yes & Yes & Yes & No & No & & NA & NA & NA & 4.26 & NA \\
\hline Case 2 & Yes & Yes & No & No & No & & NA & NA & NA & 4.32 & NA \\
\hline
\end{tabular}

${ }^{a}$ Number of the patients who had the symptom

b,c,dValues are expressed as mean for 11,8 , and 10 patients, respectively

$B C G$ Bacille Calmette-Guérin, CRP C-reactive protein, ESR erythrocyte sedimentation rate, $H b$ hemoglobin, NA not available, Plt platelet, WBC white blood cell

We performed a review of the literature using PubMed and the search terms: Kawasaki disease AND pulmonary involvement; OR Kawasaki disease AND pulmonary presentation; OR Kawasaki disease AND pleural effusion. The searches were limited to the English language and pediatric patients. Case series and single case reports involving pediatric patients with KD with pulmonary involvement were included. Inconsistencies were resolved through discussion with the author $\mathrm{SO}$, who also reviewed the literature. The authors EAA and OA searched the literature and manually screened titles and abstracts for relevance. Inconsistencies were resolved through discussion with the author SO.

Figure 2 lists the schematic analyses of the systematic literature review. At first, 25 related articles were found, but nine articles were excluded because of duplication, 
Table 2 Demographic parameters and clinical presentations of patients who had pulmonary involvement associated with Kawasaki disease

\begin{tabular}{|c|c|c|c|c|c|c|}
\hline $\begin{array}{l}\text { Authors, year, reference } \\
\text { number }\end{array}$ & Patients $(n)$ & Sex & $\begin{array}{l}\text { Age at onset of } \\
\text { disease (months) }\end{array}$ & Initial symptoms & $\begin{array}{l}\text { Fever duration } \\
\text { (days) }\end{array}$ & Chest $X$-ray findings \\
\hline Singh et al., 2018, [6] & 11 & $\begin{array}{l}\mathrm{F}, 6^{a} \\
\mathrm{M}, 5^{a}\end{array}$ & $30^{b}$ & Fever, cough, tachypnea & $14.1^{b}$ & 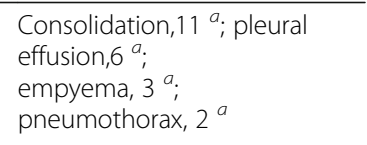 \\
\hline $\begin{array}{l}\text { Alhammadi and } \\
\text { Hendaus, 2013, [12] }\end{array}$ & 1 & $\mathrm{~F}$ & 36 & Fever, cough, sore throat & 18 & Consolidation, pleural effusion \\
\hline Lee et al., 2011, [11] & 54 & NA & NA & NA & NA & $\begin{array}{l}\text { Reticulonodular, } 17^{a} ; \\
\text { opacification, } 34^{a} ; \\
\text { consolidation, } 12^{a} \text {; pleural } \\
\text { effusion, } 5^{a} \\
\text { diffuse interstitial, } 5^{a} ; \\
\text { atelectasis, } 2^{a} ;\end{array}$ \\
\hline Lee et al., 2010, [14] & 1 & M & 22 & Fever, cough, rhinorrhea & 6 & Infiltration, pleural effusion \\
\hline Falcini et al., 2009, [15] & 1 & $\mathrm{~F}$ & 30 & Fever, cough, & 12 & Pleural effusion \\
\hline Elizabeth et al., 2007, [16] & 1 & $\mathrm{~F}$ & 36 & Fever, gum bleeding & 21 & Pleural effusion \\
\hline Yavuz et al., 2007, [17] & 1 & M & 11 & $\begin{array}{l}\text { Fever, pharyngeal erythema, } \\
\text { dyspnea }\end{array}$ & $>5$ & Pleural effusion \\
\hline $\begin{array}{l}\text { Sittiwangkul and Pongprot, } \\
2004,[13]\end{array}$ & 1 & $\mathrm{~F}$ & 11 & $\begin{array}{l}\text { Fever, jaundice, diarrhea, } \\
\text { dyspnea }\end{array}$ & 15 & Pleural effusion \\
\hline $\begin{array}{l}\text { de Magalhães et al., } \\
\text { 2012, [21] }\end{array}$ & 1 & $\mathrm{~F}$ & 3 & Fever & 10 & Infiltration \\
\hline Hamada et al., 2005, [18] & 1 & $\mathrm{~F}$ & 60 & $\begin{array}{l}\text { Fever, abdominal pain, knee } \\
\text { joint pain }\end{array}$ & 15 & Pleural effusion \\
\hline D'Souza et al., 2006, [20] & 1 & M & 5 & Fever, diarrhea, dyspnea & 7 & Pleural effusion \\
\hline Case 1 & 1 & M & 8 & Fever, sore throat & 8 & Consolidation \\
\hline Case 2 & 1 & $\mathrm{~F}$ & 11 & $\begin{array}{l}\text { Febrile seizure, sore throat, } \\
\text { cough }\end{array}$ & 14 & Consolidation \\
\hline Case 3 & 1 & $\mathrm{~F}$ & 23 & Fever, cough & 10 & Consolidation \\
\hline \multicolumn{7}{|l|}{ Freeman et al., 2003, [23] } \\
\hline Case 1 & 1 & M & 4 & Fever, cough, rash & 21 & NA \\
\hline Case 2 & 1 & M & 6 & Fever & 60 & $\begin{array}{l}\text { Normal; } \\
\text { thorax } C T \text {, pulmonary nodule }\end{array}$ \\
\hline Case 3 & 1 & NA & 5 & Fever cough, rash & 4 & $\begin{array}{l}\text { Infiltration, multiple pulmonary } \\
\text { nodules }\end{array}$ \\
\hline \multicolumn{7}{|l|}{ Kobayashi et al., 2006, [24] } \\
\hline Case 1 & 1 & $\mathrm{~F}$ & 24 & Fever, cracked lips, rash & 5 & Infiltration, pleural effusion \\
\hline Case 2 & 1 & $F$ & 24 & $\begin{array}{l}\text { Fever, cough, nasal } \\
\text { discharge }\end{array}$ & 4 & Atelectasis \\
\hline Vaidya et al.., 2017, [8] & 1 & $\mathrm{~F}$ & 3 & Fever, rash, dyspnea & 32 & $\begin{array}{l}\text { Hydropneumothorax, } \\
\text { consolidation, pneumatoceles }\end{array}$ \\
\hline \multicolumn{7}{|l|}{ Akagi et al., 2017, [19] } \\
\hline Case 1 & 1 & $\mathrm{~F}$ & 4 & $\begin{array}{l}\text { Fever, erythema of the lips, } \\
\text { rash }\end{array}$ & NA & $\begin{array}{l}\text { NA; } \\
\text { thorax MRI, bilateral multiple } \\
\text { pulmonary nodules }\end{array}$ \\
\hline Case 2 & 1 & $\mathrm{~F}$ & 5 & $\begin{array}{l}\text { Fever, erythema of the lips, } \\
\text { rash }\end{array}$ & 9 & $\begin{array}{l}\text { Infiltration; } \\
\text { thorax } C T \text {, bilateral pulmonary } \\
\text { nodules }\end{array}$ \\
\hline
\end{tabular}


non-English language, and adult age, which left 16 articles $[6,8,11-24]$. The characteristics of these patients are summarized in Tables 1, 2, and 3. Finally, 20 patients with pleural effusions due to KD were identified $[6,11-18,20$, 24]. Of the 20 reviewed patients, TTE results were available in nine patients and seven had CAI [6, 12-17, 24]. Eleven patients presented with respiratory symptoms such as cough, dyspnea, and tachypnea $[6,12-15,20]$. Only four patients $[14,17,18,24]$ had complete KD, 10 patients [6, $13,15,16,20]$ had incomplete KD, and six patients' $[11,12]$ presentations were not available. Although a definite infectious agent could be shown for two patients [18, 24], all of the patients received antibiotics except one [14]. Two patients $[6,17]$ received a second dose of IVIG, and five patients received a second dose of IVIG and corticosteroid treatment for KD [13-16, 18].

In this case, our patient initially had an exudative, noninfectious pleural effusion and no response to antibiotics. CAI was also noticed and IVIG was administered on the 15th day of fever. After IVIG treatment, our patient's clinical and laboratory findings improved dramatically, and the fever and acute-phase reactants returned to normal. It remains unclear as to whether the KD was triggered by the infection of the pleural space or if the pulmonary finding was a feature of the inflammation of KD.

Table 3 Treatment, coronary artery involvement, follow-up, and outcomes of patients who had pulmonary involvement associated with Kawasaki disease

\begin{tabular}{|c|c|c|c|c|c|}
\hline Authors, year, reference number & Infectious agent & Antibiotic treatment & CAl & Treatment & Follow-up and outcome \\
\hline Singh et al., 2018, [6] & $2^{a}$ & $11^{a}$ & $3^{a}$ & $2^{a}$, Second dose of IVIG & $9^{a}$ Normal, $2^{a} \mathrm{NA}$ \\
\hline Alhammadi and Hendaus, 2013, [12] & No & Yes & Yes & IVIG & Normal \\
\hline Lee et al., 2011, [11] & NA & NA & NA & NA & NA \\
\hline Lee et al., 2010, [14] & No & No & Yes & $\begin{array}{l}\text { Second dose of IVIG and } \\
\text { corticosteroid }\end{array}$ & Normal \\
\hline Falcini et al., 2009, [15] & No & Yes & Yes & $\begin{array}{l}\text { Second dose of IVIG and } \\
\text { corticosteroid }\end{array}$ & Normal \\
\hline Elizabeth et al., 2007, [16] & No & Yes & Yes & $\begin{array}{l}\text { Second dose of IVIG and } \\
\text { corticosteroid }\end{array}$ & Normal \\
\hline Yavuz et al., 2007, [17] & No & Yes & Yes & Second dose of IVIG & Normal \\
\hline Sittiwangkul and Pongprot, 2004, [13] & No & Yes & Yes & $\begin{array}{l}\text { Second dose of IVIG and } \\
\text { corticosteroid }\end{array}$ & $\begin{array}{l}\text { Aneurysm persisted } \\
\text { in } 2 \text { years }\end{array}$ \\
\hline de Magalhães et al., 2012, [21] & No & Yes & Yes & $\begin{array}{l}\text { Second dose of IVIG, } \\
\text { corticosteroid, MTX, and ETN }\end{array}$ & $\begin{array}{l}\text { Aneurysm decrease } \\
\text { but persisted }\end{array}$ \\
\hline Hamada et al., 2005, [18] & No & Yes & No & $\begin{array}{l}\text { Second dose of IVIG and } \\
\text { corticosteroid }\end{array}$ & Normal \\
\hline D'Souza et al., 2006, [20] & No & Yes & No & IVIG & Normal \\
\hline \multicolumn{6}{|l|}{ de Maddi et al., 2009, [22] } \\
\hline Case 1 & No & Yes & No & IVIG & Normal \\
\hline Case 2 & No & Yes & No & Not given IVIG & Normal \\
\hline Case 3 & No & Yes & No & IVIG & Normal \\
\hline \multicolumn{6}{|l|}{ Freeman et al., 2003, [23] } \\
\hline Case 1 & No & Yes & Yes & IVIG & Death \\
\hline Case 2 & NA & Yes & Yes & IVIG & Normal \\
\hline Case 3 & No & Yes & Yes & IVIG & Normal \\
\hline \multicolumn{6}{|l|}{ Kobayashi et al., 2006, [24] } \\
\hline Case 1 & Yes & Yes & NA & IVIG & Normal \\
\hline Case 2 & Yes & Yes & NA & IVIG & Normal \\
\hline Vaidya et al., 2017, [8] & No & Yes & Yes & IVIG & NA \\
\hline \multicolumn{6}{|l|}{ Akagi et al., 2017, [19] } \\
\hline Case 1 & No & No & Yes & IVIG & Normal \\
\hline Case 2 & No & Yes & Yes & IVIG & Normal \\
\hline
\end{tabular}

a Number of the patients

CAI coronary artery involvement, ETN etanercept, IVIG intravenous immunoglobulin, MTX methotrexate, NA not available 
KD can affect various systems as well as the coronary arteries, and may present with an unusual clinical picture. The diagnosis of KD with atypical presentations may be difficult for pediatricians. Early diagnosis and treatment can prevent complications.

\section{Abbreviations}

AP: Anteroposterior; CAI: Coronary artery involvement; CRP: C-reactive protein; ESR: Erythrocyte sedimentation rate; IVIG: Intravenous immunoglobulin; KD: Kawasaki disease; SAM: Sulbactam ampicillin; TTE: Transthoracic echocardiography

\section{Acknowledgements}

Not applicable.

\section{Authors' contributions}

EAA, OA, and SD drafted the initial manuscript. EAA and OA retrieved the pertinent literature. SD, YB, and SO contributed to the patient management. SO critically reviewed the manuscript. All authors have read and approved the final submitted manuscript.

\section{Funding}

No funding.

\section{Availability of data and materials}

Data sharing is not applicable to this article because no datasets were generated or analyzed during the current study.

\section{Ethics approval and consent to participate}

No ethical committee approval is required for this case report.

\section{Consent for publication}

Written informed consent was obtained from the patient's legal guardian(s) for publication of this case report and any accompanying images. A copy of the written consent is available for review by the Editor-in-Chief of this journal.

\section{Competing interests}

The authors declare that they have no competing interests.

\section{Author details}

${ }^{1}$ Department of Pediatrics, Hacettepe University Faculty of Medicine, 06100 Ankara, Turkey. ${ }^{2}$ Department of Pediatric Rheumatology, Hacettepe University Faculty of Medicine, 06100 Ankara, Turkey. ${ }^{3}$ Department of Pediatric Emergency, Hacettepe University Faculty of Medicine, 06100 Ankara, Turkey.

Received: 12 May 2019 Accepted: 9 October 2019

Published online: 25 November 2019

\section{References}

1. McCrindle BW, Rowley AH, Newburger JW, Burns JC, Bolger AF, Gewitz M, et al. Diagnosis, Treatment, and Long-Term Management of Kawasaki Disease: A Scientific Statement for Health Professionals From the American Heart Association. Circulation. 2017;135(17):e927-e99.

2. Kaman A, Aydin-Teke T, Gayretli-Aydin ZG, Oz FN, Metin-Akcan O, Eris D, et al. Two cases of Kawasaki disease presented with acute febrile jaundice. Turk J Pediatr. 2017:59(1):84-6.

3. Du Z. A case of neonatal incomplete Kawasaki disease complicated with aseptic meningitis. Zhonghua Er Ke Za Zhi. 2014;52(1):63-4.

4. Sun Q, Zhang J, Yang Y. Gallbladder Hydrops Associated With Kawasaki Disease: A Case Report and Literature Review. Clin Pediatr (Phila). 2018;57(3): 341-3.

5. Saviour MJ, Hassan S. Kawasaki Disease Presenting with Bloody Diarrhea and Acute Renal Failure: First Case. Pediatr Rep. 2017;9(2):7163.

6. Singh S, Gupta A, Jindal AK, Gupta A, Suri D, Rawat A, et al. Pulmonary presentation of Kawasaki disease-A diagnostic challenge. Pediatr Pulmonol. 2018;53(1):103-7.

7. Pilania RK, Bhattarai D, Singh S. Controversies in diagnosis and management of Kawasaki disease. World J Clin Pediatr. 2018;7(1):27-35.
8. Vaidya PC, Narayanan K, Suri D, Rohit MK, Gupta A, Singh S, et al. Pulmonary presentation of Kawasaki disease: an unusual occurrence. Int J Rheum Dis. 2017;20(12):2227-9.

9. Leahy TR, Cohen E, Allen UD. Incomplete Kawasaki disease associated with complicated Streptococcus pyogenes pneumonia: A case report. Can J Infect Dis Med Microbiol. 2012;23(3):137-9.

10. Ugi J, Lepper PM, Witschi M, Maier V, Geiser T, Ott SR. Nonresolving pneumonia and rash in an adult: pulmonary involvements in Kawasaki's disease. Eur Respir J. 2010;35(2):452-4.

11. Lee MN, Cha JH, Ahn HM, Yoo JH, Kim HS, Sohn S, et al. Mycoplasma pneumoniae infection in patients with Kawasaki disease. Korean J Pediatr. 2011;54(3):123-7.

12. Alhammadi AH, Hendaus MA. Comorbidity of Kawasaki disease and group a streptococcal pleural effusion in a healthy child: a case report. Int J Gen Med. 2013:6:613-6.

13. Sittiwangkul $R$, Pongprot $Y$. Large pleural effusion: an unusual manifestation of Kawasaki disease. Clin Pediatr (Phila). 2004;43(4):389-91.

14. Lee $\mathrm{SH}$, Jeon $\mathrm{YH}$, Yang HJ, Pyun BY. Pleural effusion and disseminated intravascular coagulopathy: the rarely reported complications of kawasaki disease. Clin Pediatr (Phila). 2010;49(6):598-600.

15. Falcini F, Vitale A, La Torre F, Conti G, Fede C, Calcagno G. Refractory pneumonia and high fever. Lancet. 2009:373(9677):1818.

16. Elizabeth KE, Ahamed MZ, Praveen KS. Atypical relapsing course of Kawasaki disease with hemorrhagic serous effusions and hepatic dysfunction. Indian Pediatr. 2007;44(10):785-7.

17. Yavuz T, Nisli K, Yilmaz C, Dindar A. Large pleural effusion necessitates chest tube drainage in a patient with Kawasaki disease. J Paediatr Child Health. 2007:43(3):191-2.

18. Hamada H, Terai M, Honda T, Kohno Y. Marked pleural and pericardial effusion with elevated Vascular Endothelial Growth Factor production: an uncommon complication of Kawasaki disease. Pediatr Int. 2005;47(1):112-4.

19. Akagi K, Abe J, Tanaka K, Tomotaki S, Iki Y, Ueda K, et al. Kawasaki disease with pulmonary nodules and coronary artery involvement: a report of two cases and a review of the literature. Int J Rheum Dis. 2017;20(11):1862-4.

20. D'Souza S, Khubchandani RP, Shetty AK. Kawasaki disease presenting with hemorrhagic pleural effusion. J Trop Pediatr. 2006;52(4):299-301.

21. de Magalhães CM, Alves NR, de Melo AV, Junior CA, Nomicronbrega YK, Gandolfi $L$, et al. Catastrophic Kawasaki disease unresponsive to IVIG in a 3month-old infant: a diagnostic and therapeutic challenge. Pediatr Rheumatol Online J. 2012;10(1):28.

22. De Maddi F, Cinelli R, Rigante D, Mazzarella G, Siani P. Lung parenchymal consolidation as an uncommon presentation and cause of delayed diagnosis in atypical Kawasaki syndrome. Rheumatol Int. 2009;29(11):1373-6.

23. Freeman AF, Crawford SE, Finn LS, Lopez-Andreu JA, Ferrando-Monleon S, Perez-Tamarit D, et al. Inflammatory pulmonary nodules in Kawasaki disease. Pediatr Pulmonol. 2003;36(2):102-6.

24. Kobayashi $Y$, Koike Y, Tokutomi T, Kuroki Y, Todoroki I. Case 2: fever, rash and pulmonary involvement. Diagnosis: Kawasaki disease. Acta Paediatr. 2006;95(9):1145-8

\section{Publisher's Note}

Springer Nature remains neutral with regard to jurisdictional claims in published maps and institutional affiliations.

Ready to submit your research? Choose BMC and benefit from:

- fast, convenient online submission

- thorough peer review by experienced researchers in your field

- rapid publication on acceptance

- support for research data, including large and complex data types

- gold Open Access which fosters wider collaboration and increased citations

- maximum visibility for your research: over $100 \mathrm{M}$ website views per year

At $\mathrm{BMC}$, research is always in progress.

Learn more biomedcentral.com/submission 